\title{
Antituberculosis Drugs Resistance and Treatment Outcomes Among Retreatment Patients in Guinea: A Five-Year Retrospective Cohort Study
}

\author{
Tamba Kallas Tonguino, ", Tamba Mina Millimouno², Boubacar Djelo Diallo ${ }^{1}$, \\ Alexandre Delamou ${ }^{2,3}$, Nimer Ortuno Gutierrez ${ }^{4}$, Mory Camara ${ }^{1}$, Boubacar Bah ${ }^{1}$, \\ Oumou Younoussa Sow ${ }^{1}$ \\ ${ }^{1}$ Faculty of Health Sciences and Techniques, Gamal Abdel Nasser University of Conakry, Conakry, Guinea \\ ${ }^{2}$ Research Unit, National Training and Research Centre in Rural Health of Maferinyah, Forecariah, Guinea \\ ${ }^{3}$ Department of Public Health, Gamal Abdel Nasser University of Conakry, Conakry, Guinea \\ ${ }^{4}$ Tuberculosis Control Project, Action Damien, Conakry, Guinea
}

Email address:

kallaskoumba@gmail.com (T. K. Tonguino)

${ }^{*}$ Corresponding author

\section{To cite this article:}

Tamba Kallas Tonguino, Tamba Mina Millimouno, Boubacar Djelo Diallo, Alexandre Delamou, Nimer Ortuno Gutierrez, Mory Camara, Boubacar Bah, Oumou Younoussa Sow. Antituberculosis Drugs Resistance and Treatment Outcomes Among Retreatment Patients in Guinea: A Five-Year Retrospective Cohort Study. Science Journal of Public Health. Vol. 7, No. 5, 2019, pp. 167-173. doi: $10.11648 /$ j.sjph.20190705.16

Received: September 9, 2019; Accepted: September 24, 2019; Published: October 11, 2019

\begin{abstract}
Tuberculosis (TB) is a global outbreak whose drug resistance is a constant threat. This study aimed at describing anti-TB drugs resistance and treatment outcomes among retreatment TB patients in Guinea between 2008 and 2012. We conducted a retrospective cohort study with a sample of 558 patients aged of at least 10 , who were admitted for TB retreatment and who were tested for anti-TB drugs susceptibility during the study period. Overall, 3187 retreatment TB patients were recorded from January 1, 2008 to December 31, 2012 in Guinea, of which 558 (17.5\%) performed susceptibility testing to antiTB drugs. We found overall resistance in 417 cases (74.7\%) including $356(85.4 \%)$ of multidrug resistance (MDR), $29(6.9 \%)$ of monoresistance (isoniazid $2.9 \%$, streptomycin $2.9 \%$, rifampicin $0.9 \%$, ethambutol $0.2 \%)$ and $32(7.7 \%$ of polydrug resistance (isoniazid + streptomycin 4.3\%, rifampicin + streptomycin $1.4 \%$, isoniazid + ethambutol + streptomycin $1.0 \%$, rifampicin + ethambutol + streptomycin 1.0\%). Most of the patients (84.6\%) with anti-TB drugs resistance were under 45 and labourers were mostly represented (27.8\%) including drivers in majority (37.9\%). MDR-TB incidence rate increased by $12.2 \%$ between $2008(65.6 \%)$ and $2012(77.8 \%)$, and the annual cure rate decreased gradually from $60.0 \%$ in 2009 to $45.7 \%$ in 2012 . Among MDR-TB patients $(\mathrm{n}=356)$, only $112(31.5 \%)$ benefited from second-line treatment regimen, of which, $51.7 \%$ were cured, $6.3 \%$ completed treatment, $24.1 \%$ died, $6.3 \%$ were lost to follow-up and $11.6 \%$ were not evaluated. The cure rate was higher in HIV-negative patients $(55.3 \%)$ than in those who were HIV-positive (35.3\%) and the death rate was the highest (41.2\%) in HIV-positive patients. Overall, treatment success rate was $58.0 \%$. This study revealed a low rate of performing drug susceptibility testing, the gradual increase of the incidence of MDR-TB each year and the gradual decrease of cure rate from year to year. Besides, anti-TB drugs resistance concerned mostly drivers in our context. Prospective studies are needed for a deep understanding of the factors associated with these persistent challenges.
\end{abstract}

Keywords: Antituberculosis, Resistance, Treatment Outcome, Retreatment Patients, Guinea

\section{Background}

Tuberculosis (TB) is a major public health issue, caused by
Mycobacterium tuberculosis [1-2]. Despite progress in decreasing TB incidence $(2 \%$ per year) and reducing mortality (about $3 \%$ per year) worldwide, $16 \%$ of patients 
died of this disease in 2017 [2]. TB is one of the top 10 causes of death and the leading cause from a single infectious agent (above HIV/AIDS) worldwide [2-3], while its treatment success is globally low (55\%) [2].

In 2014, the highest $\mathrm{TB}$ incidence rate was recorded in Asia (61\%), followed by Africa (26\%) from where came the $74 \%$ of all HIV-positive TB patients reported in the world [4]. The "World Health Organization (WHO) strategy for ending TB in 2019-2023" [5], adopted by the world health assembly in May 2014, and the epidemiological surveillance networks in heavily affected countries, represent hope for the reduction of the global burden of this disease in the context of the Sustainable Development Goal (SDG) three target three [6-7]. However, the fight against TB drug resistance remains a major public health concern worldwide.

Globally in 2017, there were an estimated 558000 new cases of rifampicin resistant TB (RR-TB), of which almost $82 \%$ were multidrug-resistant TB (MDR-TB) [2]. Inadequate treatment and non-adherence to treatment result in drug resistance in patients undergoing retreatment, especially those admitted for retreatment failure [8-10]. Retreatment TB patients represent those who have been treated previously for one month or more with anti-TB drugs and who have been diagnosed once again with the disease. These patients mainly include relapses, treatment after failure, or loss to follow-up on a first-line treatment regimen [1]. The number of these patients is not negligible. Worldwide in 2017, of the 6.7 million TB cases that were officially notified by national TB programmes to the WHO, 260,000 patients were already previously treated [2]. It has been globally estimated that $20 \%$ of previously treated tuberculosis cases would have developped MDR-TB [6].

Surveys conducted in six countries (Azerbaijan, Bangladesh, Belarus, Pakistan, South Africa and Ukraine) on resistance to all first-line anti-TB drugs (rifampicin, isoniazid, pyrazinamide and ethambutol) reported the average levels of resistance of $19 \%(95 \% \mathrm{CI}: 18-20 \%)$ in new TB cases and 43\% (95\% CI: 40-46\%) in previously treated cases [2].

Secondary resistance to Streptomycin affects $20 \%$ of global TB cases [11]. The African region recorded between 36,000 and 44,000 MDR-TB cases in 2016. Studies conducted in Benin [12] and Burkina Faso [13] on patients who had previously received TB treatment, reported almost similar rates of MDR-TB, $52.9 \%$ and $50.5 \%$, respectively.

In Guinea, studies on anti-TB drugs resistance are rare and there are no data on resistance during retreatment. The most recent official data on anti-TB drugs resistance (first-line) in Guinea dates back to 1999. However, some data on MDR-TB are recorded at the national reference laboratory of mycobacteria and at the non-government organization “Action Damien". From 2006 to 2008, an increase of MDRTB cases was noted, respectively, 17, 36 and 54 cases in 2006, 2007 and 2008 [14-15].

In order to strengthen the quality of care and follow-up of patients suffering from MDR-TB in Guinea, studies to improve the level of knowledge on anti-TB drugs resistance and treatment outcomes are necessary. Thus, the objective of this study was to describe anti-TB drugs resistance and treatment outcomes among retreatment TB patients in Guinea between 2008 and 2012.

\section{Methods}

\subsection{Study Design}

We conducted a retrospective cohort study from January 01, 2008 and December 31, 2012 (5-year period) using data from two reference sites for TB treatment in Guinea.

\subsection{Setting}

Guinea is located in West Africa with approximately 11 million inhabitants of whom $15 \%$ live in Conakry, the Capital city [16].

The pneumo-phtisiology ward of Ignace Deen national hospital (Conakry), the anti-TB reference centre of "Carrière" (Conakry) and the medical centre of the Philafrican mission in Macenta (Forest Guinea) served as study sites. The anti-TB reference centre of "Carrière" (outpatient care unit of the pneumo-phtisiology ward of Ignace Deen national hospital) and the medical centre of the Philafrican mission in Macenta are the main reference sites for TB treatment in Guinea, and they were used to collect socio-demographic and clinical data of patients. As for the pneumo-phtisiology ward of Ignace Deen, it was used to collect biological data (anti-TB susceptibility testing) through its national reference laboratory.

\subsection{Study Population}

All retreatment TB patients recorded at the study sites during the study period were considered as our study population. Specifically, patients aged of 10 and older in whom drug susceptibility testing (antibiogram or Xpert MTB/RIF) to anti-TB drugs was performed at the national reference laboratory of mycobacteria were included in the study. We excluded, new cases of TB, patients under 10 years and those whose records were not found.

\subsection{Sampling}

We carried out a non-random sampling with an exhaustive inclusion of all retreatment TB patients who performed drug susceptibility testing (antibiogram or Xpert MTB/RIF) at the national reference laboratory of Mycobacteria during the study period. The total number of retreatment cases (from which the study sample derived) for the study period were already known because all cases were recorded at the national reference laboratory of mycobacteria.

\subsection{Data Collection, Study Variables and Analysis}

Data were collected using an anonymous and standardized questionnaire. Data sources were national documents (from the national TB programme), drug susceptibility testing request forms and laboratory registers (from the national reference laboratory), medical records and MDR-TB 
patients' follow-up registers (from the two reference sites). The study variables were sociodemographic (age, sex, residence, profession), biological (Performing of drug susceptibility testing and its results), clinical (type of retreatment in patients resistant to first-line anti-TB drugs and number of MDR-TB cases per category, number of MDR-TB cases per year (to determine trends), treatment outcomes in MDR-TB patients (cured, treatment completed, died, lost to follow-up, not evaluated) depending on admission year, type of retreatment, treatment site and HIV status). Data were doubly entered to minimize errors, using EpiData Entry software, version 3.1 and analysed using SPSS software, version 21.

\subsection{Ethics Considerations}

The study was approved by the department of pneumophtisiology of the faculty of health sciences and techniques of Gamal Abdel Nasser University of Conakry. Data collection form was anonymous and identifiable only by a number.

\subsection{Operational / Standard Definitions [17]}

Overall resistance: resistance to at least one first-line antiTB drug (rifampicin, isoniazid, streptomycin, ethambutol).

Monoresistance: resistance to one first-line anti-TB drug only.

Polydrug resistance: resistance to more than one first-line anti-TB drug (other than both isoniazid and rifampicin), e.g. (rifampicin + streptomycin, isoniazid + streptomycin, isoniazid + ethambutol + streptomycin, rifampicin + ethambutol + streptomycin).

Multidrug resistance (MDR): resistance to both rifampicin and isoniazid, the two most powerful anti-TB drugs.

Extensive drug resistance: resistance to any fluoroquinolone and to at least one of three second-line injectable drugs (capreomycin, kanamycin and amikacin), in addition to MDR.

\section{Results}

\subsection{Findings of Drug Susceptibility Testing}

Overall, 3187 retreatment TB patients were notified in Guinea between 2008 and 2012, of which 558 cases (17.5\%) were tested for susceptibility to first-line anti-TB drugs at the national reference laboratory of mycobacteria. Overall resistance rate was $74.7 \%(n=417)$, including $85.4 \%(n=356)$ of MDR-TB, 6.9\% (n=29) of monoresistance and 7.7\% $(n=32)$ of Polydrug resistance. Among MDR-TB patients $(n=$ $356)$, only $112(31.5 \%)$ benefited from second-line treatment regimen (Figure 1).



Figure 1. Cascade of inclusion in the study on the resistance to anti-tuberculosis drugs among retreatment tuberculosis patients in Guinea, $2008-2012$. 


\subsection{Sociodemographic Characteristics of First-line Anti-TB Drugs Resistant Patients (N=417)}

Patients' age varied between 10 and 80 years. The age range of 25-34 was the most represented $(\mathrm{n}=151,36.2 \%)$, followed by that of $35-44(\mathrm{n}=104,24.9 \%)$. Almost $85.0 \%$ of patients were under 45 years and $67.0 \%$ were male. Two-thirds $(66.7 \%)$ of patients resided in the capital city (Conakry). The majority of resistance cases was found in labourers $(n=116,27.8)$ followed by students $(\mathrm{n}=87,20.9 \%)$ (Table 1$)$.

Table 1. Sociodemographic characteristics of retreatment tuberculosis patients resistant to first-line anti-TB drugs in Guinea, 2008-2012 (N=417).

\begin{tabular}{|c|c|c|}
\hline Variables & Number & Percentage \\
\hline \multicolumn{3}{|l|}{ Age } \\
\hline $10-24$ & 98 & 23.5 \\
\hline $25-34$ & 151 & 36.2 \\
\hline $35-44$ & 104 & 24.9 \\
\hline$\geq 45$ & 64 & 15.4 \\
\hline \multicolumn{3}{|l|}{ Sex } \\
\hline Male & 281 & 67.0 \\
\hline Female & 136 & 33.0 \\
\hline \multicolumn{3}{|l|}{ Residence } \\
\hline Conakry & 278 & 66.7 \\
\hline $\begin{array}{l}\text { Forest Guinea, Upper Guinea, Middle } \\
\text { Guinea and Lower Guinea }\end{array}$ & 122 & 29.2 \\
\hline Abroad countries $^{\mathrm{a}}$ & 17 & 4.1 \\
\hline \multicolumn{3}{|l|}{ Profession } \\
\hline Employees * & 49 & 11.8 \\
\hline Health staff & 10 & 2.4 \\
\hline Students & 87 & 20.9 \\
\hline Farmers and housewives & 67 & 16.1 \\
\hline Shopkeepers and dealers & 68 & 16.3 \\
\hline Labourers $^{\mathrm{b}}$ & 116 & 27.8 \\
\hline Unemployees & 20 & 4.8 \\
\hline
\end{tabular}

${ }^{*}=$ Person who had a paid job, except health staff.

a=Côte d'Ivoire: 10; Liberia: 3; Sierra Leone: 3; Senegal: 1

$\mathrm{b}=$ Patients who do manual work, of which 44 (37.9\%) were drivers

\subsection{Annual Trends of MDR-TB Incidence Rates $(N=356)$}

The figure 2 shows the evolution of MDR-TB incidence rates in Guinea between 2008 and 2012. Overall, MDR-TB incidence rate increased by $12.2 \%$, from $65.6 \%$ in 2008 to $77.8 \%$ in 2012 .

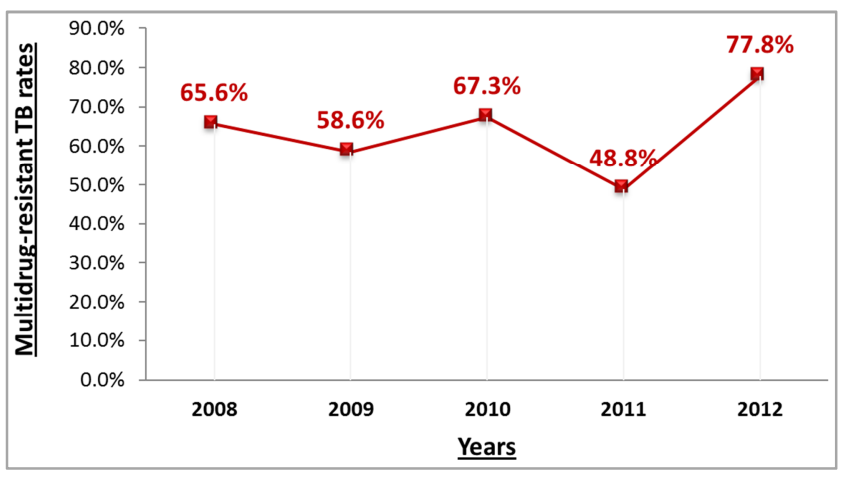

Figure 2. Annual trends of multidrug-resistant tuberculosis incidence rates among retreatment tuberculosis patients in Guinea, 2008-2012.

\subsection{Type of Retreatment in Patients Resistant to First-line Anti-TB Drugs and MDR-TB Rates (N=417)}

The type of retreatment was not mentioned for all patients $(\mathrm{N}=417)$. This information was available for only 150 patients. Among them, the majority $(n=95,63.3 \%)$ were retreated for retreatment failure, followed by relapse $(n=31$, $20.7 \%)$, failure of first-line treatment regimen $(n=20,13.3 \%)$ and loss to follow-up ( $\mathrm{n}=4,2.7 \%)$. MDR was higher among patients admitted for retreatment failure ( $n=91,60.7 \%)$ followed by those treated for relapse and treatment failure, respectively, $16 \%$ and $12.6 \%$ (Table 2 ).

Table 2. Type of retreatment in patients resistant to first-line anti-TB drugs and MDR-TB rates in Guinea, 2008-2012 (N=150)*.

\begin{tabular}{lll}
\hline Type of retreatment & $\mathbf{n ~ ( \% )}$ & $\begin{array}{l}\text { MDR-TB } \\
\text { n (\%) }\end{array}$ \\
\hline Treatment failure (first-line regimen) & $20(13.3)$ & $19(12.6)$ \\
Relapse & $31(20.7)$ & $24(16.0)$ \\
Loss to follow-up & $4(2.7)$ & $4(2.7)$ \\
Retreatment failure (second-line regimen) & $95(63.3)$ & $91(60.7)$ \\
Total & $150(100.0)$ & $138(92.0)$ \\
\hline
\end{tabular}

*Information about the type of retreatment in patients resistant to first-line anti-TB drugs were available for only 150 patients, otherwise $\mathrm{N}$ should be 417.

\subsection{Treatment Outcomes in MDR-TB Patients $(N=112)$}

The table 3 depicts treatment outcomes in the 112 MDRTB patients who benefited from second-line treatment regimen. Among these patients, 51.7\% were cured, $6.3 \%$ completed treatment, $24.1 \%$ died, $6.3 \%$ were lost to followup and $11.6 \%$ were not evaluated. Overall, treatment success rate (cured + treatment completed) was $58.0 \%$. While the number of MDR-TB patients increased from 2009 to 2012 , the annual cure rate decreased gradually from $60.0 \%$ in 2009 to $45.7 \%$ in 2012 . A little over half $(55.0 \%)$ of MDR patients who were treated for failure of second-line regimen treatment were cured; in contrast, they constituted the group which recorded the highest death rate $(27.5 \%, 22 / 80)$. However, patients treated for relapse were cured in $52.9 \%$ of cases without any death and half $(50.0 \%)$ of those treated for failure of first-line treatment regimen were cured, with only one death recorded in this group $(1 / 8,12.5 \%)$. Treatment success rates by treatment site were $67.7 \%$ for the anti-TB reference centre in Conakry and $44.7 \%$ for the medical centre of Philafrican mission in Macenta. The overall cure rate was higher in HIV-negative patients $(55.3 \%)$ than in those who were HIV-positive $(35.3 \%)$ and the mortality rate was the highest $(41.2 \%)$ in HIV-positive patients. 
Table 3. Treatment outcomes in multidrug-resistant tuberculosis patients at two treatment sites ${ }^{l}$ in Guinea, 2008-2012 (N=112).

\begin{tabular}{|c|c|c|c|c|c|c|}
\hline \multirow[t]{2}{*}{ Variables } & Cured & $\begin{array}{l}\text { Treatment } \\
\text { completed }\end{array}$ & Died & $\begin{array}{l}\text { Lost to follow- } \\
\text { up }\end{array}$ & Not evaluated & Total \\
\hline & n (\%) & n (\%) & n (\%) & n (\%) & n (\%) & n (\%) \\
\hline \multicolumn{7}{|l|}{ Admission year } \\
\hline 2008 & $12(57.1)$ & $4(19.0)$ & $4(19.0)$ & $0(0.0)$ & $1(4.8)$ & $21(18.8)$ \\
\hline 2009 & $6(60.0)$ & $2(20.0)$ & $2(20.0)$ & $0(0.0)$ & $0(0.0)$ & $10(9.0)$ \\
\hline 2010 & $12(54.5)$ & $0(0.0)$ & $8(36.4)$ & $2(9.1)$ & $0(0.0)$ & $22(19.6)$ \\
\hline 2011 & $12(50.0)$ & $0(0.0)$ & $8(33.3)$ & $3(12.5)$ & $1(4.2)$ & $24(21.4)$ \\
\hline 2012 & $16(45.7)$ & $1(2.9)$ & $5(14.3)$ & $2(5.7)$ & $11(31.4)$ & $35(31.2)$ \\
\hline Total & $58(51.7)$ & $7(6.3)$ & $27(24.1)$ & $7(6.3)$ & $13(11.6)$ & $112(100.0)$ \\
\hline \multicolumn{7}{|l|}{ Type of retreatment } \\
\hline Treatment failure (first-line regimen) & $4(50.0)$ & $1(12.5)$ & $1(12.5)$ & $1(12.5)$ & $1(12.5)$ & $8(7.1)$ \\
\hline Relapse & $9(52.9)$ & $0(0.0)$ & $3(17.6)$ & $1(5.9)$ & $4(23.5)$ & $17(15.2)$ \\
\hline Loss to follow-up ${ }^{2}$ & $1(25.0)$ & $0(0.0)$ & $0(0.0)$ & $1(25.0)$ & $2(50.0)$ & $4(3.6)$ \\
\hline Treatment failure (second-line regimen) & $44(55.0)$ & $6(7.5)$ & $22(27.5)$ & $4(5.0)$ & $4(5.0)$ & $80(71.4)$ \\
\hline Not determined $^{3}$ & $0(0.0)$ & $0(0.0)$ & $1(33.3)$ & $0(0.0)$ & $2(66.7)$ & $3(2.7)$ \\
\hline \multicolumn{7}{|l|}{ Treatment site } \\
\hline Antituberculosis reference centre & $39(60.0)$ & $5(7.7)$ & $15(23.1)$ & $6(9.2)$ & $0(0.0)$ & $65(58.0)$ \\
\hline \multicolumn{3}{|l|}{ HIV status } & $12(25.5)$ & $1(2.1)$ & $13(27.7)$ & $47(42.0)$ \\
\hline HIV (+) & $6(35.3)$ & $1(5.9)$ & $7(41.2)$ & $3(17.6)$ & $0(0.0)$ & $17(15.2)$ \\
\hline HIV (-) & $52(55.3)$ & $6(6.4)$ & $19(20.2)$ & $4(4.3)$ & $13(13.8)$ & $94(83.9)$ \\
\hline Not determined ${ }^{4}$ & $0(0.0)$ & $0(0.0)$ & $1(100.0)$ & $0(0.0)$ & $0(0.0)$ & $1(0.9)$ \\
\hline
\end{tabular}

${ }^{1}$ Antituberculosis reference centre located at "Carrière" in Conakry and medical centre of Philafrican mission in Macenta; ${ }^{2}$ Patients treated after being lost to follow-up; ${ }^{3}$ Type of retreatment was unknown; ${ }^{4} \mathrm{HIV}$ status not mentioned.

\section{Discussion}

To our knowledge, this study is the first in Guinea to describe anti-TB drugs resistance and treatment outcomes among retreatment TB patients. Hence, it showed some major challenges in managing TB including low rate of performing drug susceptibility testing (about one out of six patients), low rate of MDR-TB patients who benefited from second-line treatment regimen (only one-third) and the gradual decrease of cure rate in MDR-TB patients each year since 2009. This could explain the increase of anti-TB resistance in the country especially MDR from 2006 to 2008 [14-15] and as we observed in the current study (2008-2012).

The high incidence of MDR-TB (eight out of ten) found in our study is widely above the literature data [12-13]. This could be attributed to the delay in referring TB clinically suspected cases to TB management services and the mismanagement of patients who are susceptible to anti-TB drugs, knowing that more than half of retreatment TB patients in our study were cases of first-or second-line treatment regimen failure.

Patients under 45 years were identified as the target of the disease in our study, eight out of ten. These findings were similar to those found in several studies [12, 18-19]. The predilection of MDR-TB for this more active population mostly drivers in our context, regularly carrying out high promiscuity and the high rate of retreatment failure should worry and lead health authorities and stakeholders involved in fighting $\mathrm{TB}$ to set-up new and effective strategies or interventions.

The higher proportion of labourers (more than one-third) could be explained by the low level of education and the poor economic status of this socio-professional category, making it one of groups the most at risk of TB.

Overall, the incidence of MDR-TB increased by $12.2 \%$ between 2008 and 2012. This could be justified by the insufficiency of qualified healthcare providers and the use of a single and standardized treatment regimen for all patients $(n=112)$ who benefited from treatment; and this despite the considerable involvement of "Action Damien". It is known that an individualized treatment regimen for MDR-TB could increase the cure rate and minimize possible resistance cases and deaths.

A study conducted in Algeria in 2012 reported a higher cure rate with an individualized treatment regimen $(73 \%)$ compared to standardized treatment regimen (42\%) [20].

The overall treatment success rate found in our study was lower (about six out of ten patients) than that (about nine out of ten patients) reported in a five-year retrospective cohort study conducted in Ethiopia between 2009 and 2014 [19]. In the Ethiopian context, Directly Observed Therapy-Short course (DOTS) was implemented as the standard of care [21]. Patients were observed swallowing each dose of TB medications in front of healthcare providers for the first two months and care attendant at home for the remaining months of treatment [19].

In Guinea, individualized treatment regimen and strategies such as DOTS should be encouraged for particularly MDRTB patients in order to reduce the incidence of MDR-TB and improve the cure rate and treatment success. Besides, it is necessary to strengthen the diagnosis of TB cases by making available susceptibility testing for all patients before undergoing any treatment, and all those diagnosed with MDR should accessed to treatment. Finally, particular attention 
should be paid to the quality and compliance of the prescribed treatment and this required continuous capacity building of both healthcare providers and laboratory staff, as well as awareness campaigns to improve the level of knowledge and attitudes of the population and even health staff about this outbreak.

The strengths of this study were its national coverage including the two main reference sites for TB treatment and the single national reference laboratory, during a sufficiently long period. The study report also followed Strengthening the Reporting of Observational Studies in Epidemiology (STROBE) guidelines [22]. Major limitations were the low rate of performing drug susceptibility testing in patients and the lack of some information in MDR-TB patients such as the type of retreatment and extensive drug-resistance (XDR). Moreover, some patients $(11.6 \%)$ were not evaluated and others were lost to follow-up (6.3\%).

\section{Conclusion}

In Guinea, despite the availability of anti-TB drugs and their susceptibility testing, new techniques for the diagnosis of MDR-TB and trained health staff, the management of TB remains a potential concern. This study revealed major challenges including a low rate of performing drug susceptibility testing, the gradual increase of the incidence of MDR-TB each year and the gradual decrease of cure rate from year to year. Besides, some patients were retreated after being lost to follow-up, whereas, the type of retreatment and the HIV status were not known for all patients. Healthcare providers should exhaustively perform anti-TB drugs susceptibility testing in patients, while carrying out HIV screening testing before starting anti-TB treatment, and ensure their individual close follow-up for better treatment outcomes. The findings of this study should enable health authorities to set up more effective strategies and strong recommendations in order to successfully care for TB patients and eradicate the Mycobacterium tuberculosis in the country. Moreover, qualitative studies are needed for a deep understanding of the factors associated with the persistent current issues in managing TB in Guinea.

\section{Authors' Contributions}

All authors listed, have made substantial, direct and intellectual contribution to the work, and approved it for publication.

\section{Conflict of Interest Statement}

All authors declare that there are no conflicts of interest

\section{Acknowledgements}

We sincerely thank the managers and staffs of the pneumophtisiology ward of Ignace Deen national hospital (specifically the national reference laboratory of mycobacteria), the anti-TB reference centre of "Carrière" and the medical centre of the Philafrican mission in Macenta.

\section{References}

[1] Organisation Mondiale de la Santé (OMS). Mettre fin à la tuberculose d'ici 2030: cadre pour la mise en œuvre de la "stratégie de l'OMS pour mettre fin a la tuberculose" dans la région africaine au cours de la période 2016 - 2020. OMS, Bureau régional de l'Afrique. 2017.

[2] World Health Organization, Global Tuberculosis Report 2018, WHO/CDS/TB/2018.20. World Health Organization, Geneva, Switzerland, 2018. Available at: https://www.who.int/tb/publications/global_report/en/; Accessed on August 16, 2019.

[3] Organisation Mondiale de la Santé, Rapport sur la lutte contre la tuberculose dans le monde en 2017: Résumé d'orientation. Génève, 2017.

[4] Meyssonnier V. Epidémiologie de la tuberculose et de la résistance aux antituberculeux. Université Pierre et Marie Curie-Paris VI; 2012 [cited 2016 May 19]. Available from: https://tel.archives-ouvertes.fr/tel-00833269/

[5] Organisation Mondiale de la Santé. Tuberculose (TB): Impact mondial de la tuberculose. OMS, Bureau régional de l'Afrique. 2017

[6] World Health Assembly. Resolution A67/11. United Nations. 2014.

[7] Organisation Mondiale de la Santé (OMS). Rapport sur la lutte contre la tuberculose dans le monde. OMS, Genève, 2013. Google Sch.

[8] World Health Assembly. Resolution A/RES/71/4. United Nations. 2018. Available at: http://apps.who.int/gb/ebwha/pdf_files/WHA71/A71_4en.pdf?ua $=1$

[9] OMS Rapport sur la lutte contre la tuberculose dans le monde. 2011. WHO/HTM/TB/2011.16. Disponible sur (dernier accès: 05; 2012).

[10] Veziris N, Jarlier V and Robert J. La résistance aux antituberculeux en France en 2009 2010. Bull Épidemiologique Hebdomadair. 2012; 24: 25.

[11] Veziris N and Robert J. Résistance aux antituberculeux et impasse thérapeutique. MS Médecine Sci. 2010; 26 (11): 97680 .

[12] Ade S, Adjibodé O, Wachinou P, Toundoh N, Awanou B and Agodokpessi G. Characteristics and Treatment Outcomes of Retreatment Tuberculosis Patients in Benin. Hindawi Publishing Corporation Tuberculosis Research and Treatment Volume 2016, Article ID 1468631, 7 pages http://dx.doi.org/10.1155/2016/1468631

[13] Sangaré L, Diandé S, Badoum G, Dingtoumda B and Traoré AS. Résistance aux antituberculeux chez les cas de tuberculose pulmonaire nouveaux ou traités antérieurement au Burkina Faso. Int J Tuberc Lung Dis. 2010; 14 (11): 1424-9.

[14] World Health Organization. Global tuberculosis report 2015. Geneva, Switzerland: World Health Organization; 2015.

[15] Ministère de la Santé. Guide de prise en charge de la tuberculose pharmacorésistante en Guinée. PNLAT; 2012. 
173 Tamba Kallas Tonguino et al:: Antituberculosis Drugs Resistance and Treatment Outcomes Among Retreatment Patients in Guinea: A Five-Year Retrospective Cohort Study

[16] Institut National de la Statistique, Récensement Général de la Population et de l'Habitation (RGPH). Guinée: Institut National de la Statistique. Avril 2014. Available at: http://www.ins.ci/n/documents/RGPH2014_expo_dg.pdf. Accessed on: $15^{\text {th }}$ April 2018.

[17] World Health Organization, "Definitions and reporting framework for tuberculosis_2013 revision," Tech. Rep.WHO/HTM/TB/2013.2, World Health Organization, Geneva, Switzerland, 2013, http://apps.who.int/iris/bitstream/10665/79199/1/9789241505 345eng.pdf.

[18] Nabukenya-Mudiope MG, Kawuma HJ, Brouwer M, Mudiope $\mathrm{P}$ and Vassall A Tuberculosis retreatment 'others' in comparison with classical retreatment cases; a retrospective cohort review. BMC Public Health (2015) 15: 840.

[19] Getnet F, Sileshi H, Seifu W, Yirga S and Alemu AS. Do retreatment tuberculosis patients need special treatment response follow-up beyond the standard regimen? Finding of five-year retrospective study in pastoralist setting. Getnet et al. BMC Infectious Diseases (2017) 17: 762.

[20] Ouardi A, Hadjadj M, Bentata K and Berrabah Y. Analyse des résultats de traitement de la tuberculose multirésistante (TBMR) en fonction du régime thérapeutique prescrit. Rev Mal Respir. 2013; 30: A169.

[21] Sharma SK, Mohan A. Directly observed treatment, shortcourse (DOTS). JIACM. 2004; 5 (2): 109-13.

[22] E. Von Elm, D. G. Altman, M. Egger, S. J. Pocock, P. C. Gøtzsche and J. P. Vandenbroucke, The Strengthening the Reporting of Observational Studies in Epidemiology (STROBE) statement: guidelines for reporting observational studies. Lancet 2007; 370: 1453-1457. 